\title{
NOUVELL
}

\section{Contrôle de la localisation de la protéine kinase $A$, une propriété des AKAP partagée par une protéine précoce des adénovirus}

Mériem Imarazène ${ }^{1}$, Ouidad Aouragh ${ }^{1}$, Karim Benihoud ${ }^{2}$
${ }^{1} M 1$ Biologie Santé, Université Paris-Saclay, 91405 Orsay, France ;

${ }^{2}$ Vectorologie et thérapeutiques anticancéreuses, UMR 8203, CNRS, Université Paris-Sud, Institut Gustave Roussy, Université Paris-Saclay, 94800 Villejuif, France.

imarazene.meriem@gmail.com widedaouragh@gmail.com karim.benihoud@u-psud.fr

vecteurs codant les sous-unités régulatrices ( $R \mid \alpha$ et $R \mid l \alpha$ ) et catalytiques $(C \alpha)$ de la PKA, et des protéines $\varepsilon l A$ provenant de différents sérotypes d'Ad humains. Puis, par des techniques de co-immunoprécipitation, ils ont montré que les protéines $\varepsilon l A$ issues des sérotypes $3,5,9,12$ et 40 (mais pas des sérotypes 4 et 52 ) étaient capables d'interagir avec les sous-unités RI $\alpha$ et RII $\alpha$. De plus, ils ont montré que, quand il y avait interaction, celle-ci se faisait via la liaison d'un motif d'une dizaine de résidus dans la partie amino-terminale des protéines $\varepsilon l A$ avec le domaine $D / D$ des sous-unités régulatrices $R I \alpha$ et $\mathrm{RI} \alpha$.

Relocalisation nucléaire des sousunités régulatrices de la PKA par les protéines $\varepsilon 1 A$ de certains sérotypes adénoviraux

Pour étudier l'impact de l'interaction des protéines $\varepsilon l A$ sur la localisation intracellulaire des sous-unités RI $\alpha$ et RII $\alpha$, les auteurs ont ensuite utilisé des techniques d'immunofluorescence et d'imagerie cellulaire. L'analyse des cellules HeLa exprimant différentes protéines $\varepsilon l A$ (à la suite de la transfection par des plasmides ou l'infection par différents adénovirus) a permis de répartir les protéines $\varepsilon l A$ en 3 groupes: des protéines $\varepsilon l A$ (Ad4, Ad40 et Ad52) incapables de relocaliser les sous-unités régulatrices du cytoplasme vers le noyau, des protéines $\varepsilon l A$ capables de reloca- 


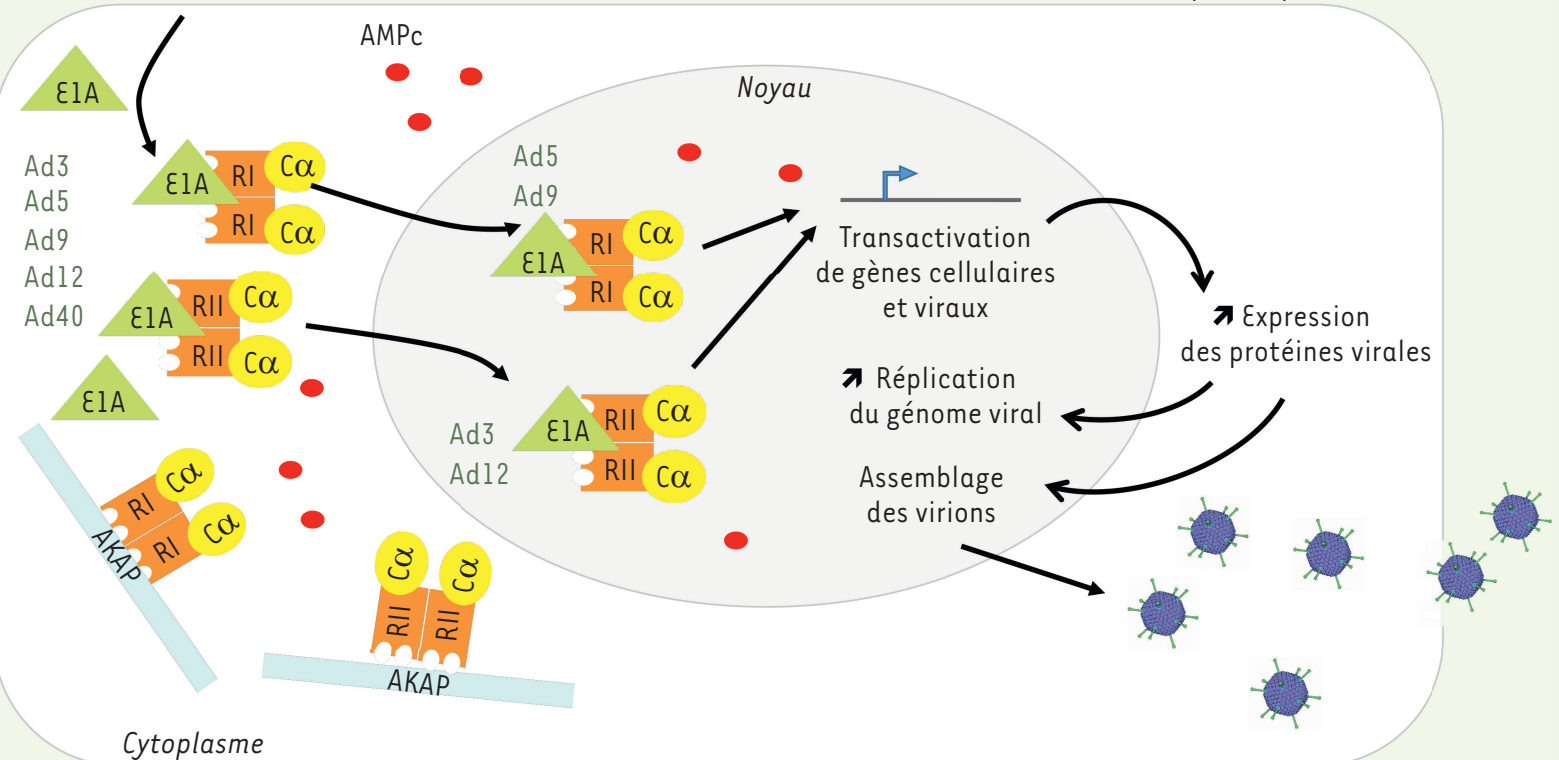

Figure 1. Mimétisme moléculaire par les protéines $\varepsilon 1 A$ des adénovirus des protéines AKAP. Les protéines $\varepsilon 1 A$ sont les premières protéines de l'adénovirus exprimées après l'infection des cellules. Les protéines $\varepsilon 1 A$ de nombreux sérotypes viraux (sérotypes 3, 5, 9, 12 et 40) sont capables de mimer les fonctions d'une AKAP cellulaire en interagissant avec les sous-unités régulatrices de la PKA. Les protéines $\varepsilon 1 A$ des Ad5 et Ad9 interagissent avec la sous-unité catalytique RI, tandis que les protéines $\varepsilon 1$ A des sérotypes 3 et 12 interagissent préférentiellement avec les sous-unités catalytiques RII. II en résulte un import nucléaire de la PKA qui conduit à une transactivation par cette protéine des gènes cellulaires et viraux, à une augmentation de la synthèse des protéines virales et de la réplication du génome viral permettant une meilleure production virale. AMPc: adénosine monophosphate cyclique. Le pictogramme de l'Ad est issu de https://pdbl01.rcsb.org/motm/132

liser spécifiquement la sous-unité RI $\alpha$ (Ad5 et Ad9) ou la sous-unité RII $\alpha$ (Ad12 et $A d 3)$. Dans le cas particulier de l'Ad5, les expériences ont révélé que cette relocalisation nécessitait le site de localisation nucléaire présent dans la région carboxy-terminale de la protéine $\varepsilon l A$. Enfin, l'étude de protéines $\varepsilon l A$ chimères entre la protéine $\varepsilon l A$ de l'Ad5, capable de fixer RI $\alpha$ mais pas RII $\alpha$, et la protéine $\varepsilon l A$ de l'Ad4, incapable de fixer RI $\alpha$ mais fixant RII $\alpha$, a démontré que le domaine amino-teminal des protéines $\varepsilon 1 A$ dictait la spécificité d'interaction avec les sous-unités régulatrices et, par voie de conséquence, la localisation de ces sous-unités. II suffit de muter quelques résidus de cette région amino-terminale des protéines $\varepsilon l A$ pour perdre l'interaction avec les sous-unités régulatrices.

\section{L'interaction des protéines $\varepsilon 1 A$ avec} la PKA, une étape indispensable à la production de différents sérotypes adénoviraux

Les chercheurs ont ensuite voulu comprendre l'intérêt fonctionnel de l'interaction entre les protéines $\varepsilon l A$ et les sous-unités régulatrices de la PKA. IIs ont tout d'abord montré que l'expression d'un gène rapporteur sous le contrôle d'un promoteur répondant à l'AMPc était induite dans des cellules exprimant les protéines $\varepsilon l A$ de différents sérotypes, mais pas lorsqu'on exprimait des protéines $\varepsilon l A$ mutées pour le domaine d'interaction avec les sous-unités régulatrices de la PKA. Ceci démontrait l'importance de l'interaction entre $\varepsilon l A$ et les sous-unités régulatrices pour le déclenchement de l'activité transactivatrice de la PKA sur les promoteurs qu'elle contrôle. De plus, l'inhibition des sous-unités régulatrices ou/et catalytiques de la PKA par interférence ARN dans les cellules infectées par différents sérotypes adénoviraux conduit à une diminution de la réplication du génome viral et de la production des virions. Ces résultats soulignent l'importance de l'activité PKA et donc de l'interaction entre $\varepsilon l A$ et la sous-unité régulatrice dans le cycle infectieux des adénovirus humains. Ils sont en accord avec l'étude précédente du même groupe montrant, dans le cas de l'Ad5, que l'interaction عlA-PKA était nécessaire à la transcription des gènes viraux précoces [3]. Il faut noter cependant que, dans la nouvelle étude, l'inhibition des sousunités catalytiques réduit la réplication virale de tous les sérotypes adénoviraux testés, y compris l'Ad4 dont la protéine عlA n'interagit pas avec les sous-unités régulatrices de la PKA. Ceci suggère qu'à 
côté d'une activité PKA mobilisée de manière dépendante de la protéine $\varepsilon l A$, il existe une activité PKA mobilisée indépendamment des protéines $\varepsilon 1 A$.

Les données de cette étude [4], combinées aux travaux précédents de la même équipe [3], démontrent que l'interaction des protéines $\varepsilon l A$ de l'adénovirus avec la PKA est une caractéristique commune à de nombreux sérotypes adénoviraux. Les résultats obtenus ont révélé un mécanisme moléculaire original fondé sur l'interaction des protéines $\varepsilon l A$ avec le domaine $D / D$ des sous-unités régulatrices de la PKA via un motif similaire à celui des AKAP cellulaires. Ainsi, ces travaux démontrent pour la première fois l'existence d'AKAP virales et ajoutent une nouvelle fonction à celles déjà identifiées pour les protéines દlA comme l'interaction avec la protéine $\mathrm{Rb}$ (rétinoblastome) [5] ou la modulation du programme épigénétique [6] de la cellule hôte. De manière plus générale, l'étude de la façon dont les protéines virales s'intègrent dans les réseaux de signalisation des cellules hôtes permet de mieux comprendre comment les virus prennent le contrôle de ces cellules pour créer un environnement propice à leur réplication. $\diamond$

Mimicry of cellular A kinase anchoring proteins, a conserved function of $\varepsilon l A$ early proteins shared by several human adenovirus species

\section{LIENS D'INTÉRÊT}

Les auteurs déclarent n'avoir aucun lien d'intérêt concernant les données publiées dans cet article.

\section{RÉFÉRENCES}

1. Sample V, DiPilato LM, Yang JH, et al. Regulation of nuclear PKA revealed by spatiotemporal manipulation of cyclic AMP. Nat Chem Biol $2012 ; 8$ : 375-82.

2. Torres-Quesada 0 , Mayrhofer JE, Stefan $\varepsilon$. The many faces of compartmentalized PKA signalosomes. Cell Signal $2017 ; 37: 1-11$

3. King CR, Cohen MJ, Fonseca GJ, et al. Functional and structural mimicry of cellular protein kinase A anchoring proteins by a viral oncoprotein. PLOS Pathog 2016; 12 : e1005621.

4. King CR, Gameiro SF, Tessier TM, et al. Mimicry of cellular $\mathrm{A}$ kinase-anchoring proteins is a conserved and critical function of $\varepsilon 1 A$ across various human adenovirus species. J Virol 2018 ; 92 : e01902-17.

5. Whyte P, Buchkovich KJ, Horowitz JM, et al. Association between an oncogene and an anti-oncogene: the adenovirus $\varepsilon 1 \mathrm{~A}$ proteins bind to the retinoblastoma gene product. Nature $1988 ; 334: 124-9$.

6. Horwitz GA, Zhang K, McBrian MA, et al. Adenovirus small ela alters global patterns of histone modification. Science $2008 ; 321: 1084-5$

\section{www.myobase.org}

Catalogue en ligne disponible gratuitement sur Internet publié par l'AFM-Téléthon. Retrouvez facilement toutes les références bibliographiques sur les maladies neuromusculaires, les situations de handicap qu'elles génèrent et leurs aspects psychologiques.

Myobase donne un accès libre à $75 \%$ du fonds documentaire collecté depuis 1990 , représentant plus de 40000 références spécifiques du domaine des maladies neuromusculaires.

$>$ articles de la littérature

biomédicale

et psycho-sociale

\section{$>$ livres, thèses}

$>$ guides d'associations et rapports institutionnels d'agences internationales

$>$ brèves en français, synthèses des articles médico-scientifiques internationaux les plus pertinents

\section{$>$ publications}

AFM-Téléthon destinées aux professionnels de santé ou aux personnes atteintes de maladie neuromusculaire et à leur entourage
UN OUTIL ERGONOMIQUE, UNE INTERFACE BILINGUE

- Laissez-vous guider par les tutoriels

- Lancez une recherche et affinez votre sélection grâce aux filtres

TOUT MYOBASE
Rechercher...
Recherche avancée
Histo
\begin{tabular}{ll} 
FILTRES \\
Type de document \\
$\square$ Article [3443] \\
$\square$ Publication AFM [176] \\
$\square$ Thèse/Mémoire [107] \\
$\square$ Brève [102] \\
\hline
\end{tabular}

\section{$\checkmark$ PUBLICATIONS AFM-Téléthon}

\section{$\checkmark$ BRÈVES}

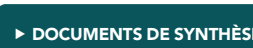

$\triangleright$ DOCUMENTS DE SYNTHĖSE

INSTITUT DES BIOTHÉRAPIES PUBLICATIONS

- Partagez les résultats de votre recherche

\section{UN ACCÈS facile et simple}

Rechercher avec des opérateurs :

- guillemets pour une expression "maladie de pompe"

- + pour signifier ET, et retrouver tous les documents contenant les deux mots "fauteuil +électrique"

- - pour signifier NON et enlever le mot de la recherche

"autonomie -établissement"

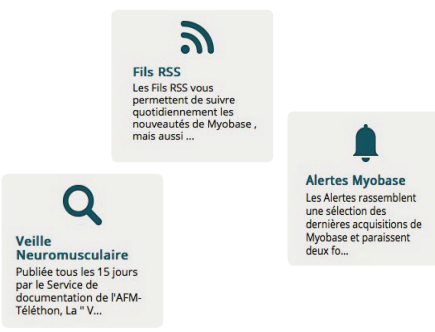

- Cliquez sur l'onglet

thématique qui vous convient (haut de la page d'accueil)

- Créez vos alertes personnalisées en ouvrant un compte personnel

- Téléchargez la Veille Neuromusculaire

- Abonnez-vous aux flux RSS 